\title{
Wie funktioniert Riechen?
}

\author{
Andreas Keller
}

\section{Zusammenfassung}

Die Klonierung der ersten Duftrezeptoren Anfang der 90er Jahre und die dadurch ermöglichten Experimente haben immens zum Verständnis der biologischen Grundlagen des Geruchssinns beigetragen. Ein Organismus exprimiert eine große Anzahl verschiedener Duftrezeptoren. Die allgemein akzeptierte Vorstellung ist, dass jede Riechzelle nur einen Duftrezeptor verwendet. Ein bestimmter Geruchseindruck entsteht dadurch, dass ein Duftmolekül an eine Kombination von Duftrezeptoren bindet und dadurch eine duftspezifische Kombination von Riechzellen aktiviert. Es wird vermutet, dass die Interaktion eines Duftmoleküls mit einem Rezeptor von der Form des Moleküls abhängt. In diesem Übersichtsartikel sollen die Ergebnisse psychophysikalischer Experimente und physiologischer Untersuchungen über den Geruchssinn vorgestellt werden. Besonderer Wert wird dabei auf Befunde gelegt, die mit dem aktuellen Modell nicht ohne weiteres zu erklären sind.

\begin{abstract}
How does the sense of smell work?

The cloning of the first olfactory receptor genes in the early 90s and the new experimental designs based on it contributed greatly to the understanding of the biological basis of the sense of smell. An organism expresses many different olfactory receptor genes. The widely accepted idea is that each olfactory sensory neuron uses only one olfactory receptor. A certain odor impression results from the binding of the odor molecule to a combination of olfactory receptors and consequential activation of a set of olfactory sensory neurons. The interaction of a molecule and the receptor is believed to depend on the shape of the odor molecule. In this review article psychophysical experiments and physiological studies concerning the sense of smell are discussed with a special emphasis on findings that can not be easily explained by the current model.
\end{abstract}

Key words: olfaction; odorant receptor; smell; olfactory code

\section{Einleitung}

Düfte faszinieren die Menschen seit jeher und die psychologische Forschung macht immer deutlicher, wie stark unser Verhalten und Befinden unbewusst von Gerüchen beeinflusst wird. Trotzdem ist das Verständnis der biologischen Grundlagen des Riechens weit hinter dem der anderen Sinne zurückgeblieben. Dies liegt zum einen an der Komplexität des Geruchssinns. Unsere Farbwahrnehmung beispielsweise wird durch drei verschiedene Typen von Photorezeptoren vermittelt, während sich im menschlichen Genom etwa 350 verschiedene Gene für Duftrezeptoren finden (Zozulya et al. 2001). Darüber hinaus ist es nicht möglich, Geruchsreize zu quantifizieren (Exkurs: Eine Systematik der Gerüche). Während eine Farbe über die Wellenlänge des Lichts und ein Ton über seine Frequenz definiert sind, unterscheiden sich Duftmoleküle in mehreren, schwer quantifizierbaren Variablen.
Die Grundlage für neue experimentelle Ansätze, die zu einem besseren Verständnis der biologischen Grundlagen des Geruchssinns führten, wurde 1991 mit der Identifizierung und Klonierung der ersten Duftrezeptoren geschaffen (Buck und Axel 1991). Duftrezeptoren sind membrangebundene Rezeptoren, die in Riechzellen exprimiert werden. Duftrezeptoren interagieren mit verschiedenen Molekülen und signalisieren induziert durch diese Interaktion dem Gehirn die Anwesenheit eines bestimmten Duftmoleküls in der Luft. Es gibt eine große Anzahl verschiedener Duftrezeptoren, die mit verschiedenen Duftmolekülen interagieren. Ein Duftmolekül bindet an eine Kombinationen von Duftrezeptoren, womit der für das Molekül typische Geruchseindruck entsteht (Abbildung 1).

\section{Der Geruch eines Moleküls}

Beim Rasenmähen wird cis-3-Hexen-1-ol freigesetzt, der Geruch von frisch geschnit- tenem Gras. Dieser Geruch und andere Düfte, die nach Gras, Blätter oder Grünpflanzen riechen, werden von Parfümeuren als „grün“ bezeichnet. Cis-3-Hexen-1-ol ist ein Alkohol mit sechs C-Atomen, einer Alkoholgruppe an Position 1 und einer Doppelbindung an Position 3. Die Düfte von Hexen-1-olen mit der Doppelbindung an anderer Position sowie die Düfte der entsprechenden Aldehyde unterscheiden sich von dem Gras-Geruch von cis-3-Hexen-1-ol, fallen aber größtenteils auch in die Kategorie „grün“. Die Düfte von Molekülen, die strukturell ähnlich sind, sind ähnlicher als die von strukturell weniger ähnlichen Molekülen (Hatanaka et al. 1992). Interessanterweise haben andere „grün“ riechende Moleküle keine offensichtlichen strukturellen Gemeinsamkeiten (Abbildung 3a). Alkohole und Aldehyde und sogar Moleküle ohne funktionelle Gruppen, gehören dieser Duftkategorie an. Auch in ihrer Größe und Form variieren ,grüne“ Duftmolekül erheblich. Warum riechen sie also ähnlich, wenn der Geruch eines Moleküls durch dessen Form und der dadurch resultierenden Kombination von aktivierten Riechzellen bestimmt wird?

Es gibt viele Beispiele von Molekülen, bei denen Struktur und Geruch ähnlich sind. Ein

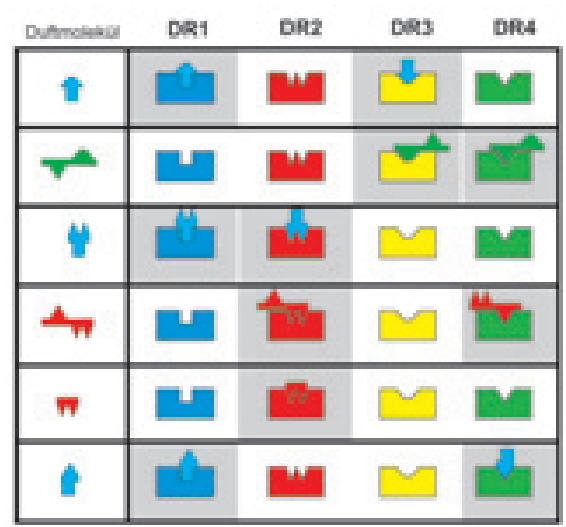

Abb. 1: Der olfaktorische Code Ein Duftmolekül wird von einem bestimmten Repertoire an Duftrezeptoren erkannt. Jeder Duftrezeptor interagiert nur mit einem Teil des Moleküls. Verschiedene Rezeptoren interagieren mit verschiedenen Teilen des Duftmoleküls, so genannten Odotopen. Das Aktivierungsmuster der Rezeptoren vermittelt den für das Molekül typischen Geruchseindruck. In dem hier gezeigten Beispiel mit vier verschiedenen Duftrezeptoren könnten demnach 15 verschiedene Duftmoleküle unterschieden werden. Tatsächlich haben manche Arten über 1000 verschiedene Duftrezeptoren und sind somit in der Lage, zumindest theoretisch eine sehr große Zahl von Gerüchen zu unterscheiden (nach Malnic et al. 1999). 


\section{Exkurs I}

\section{Eine Systematik der Gerüche}

Seit jeher wird versucht, die scheinbar unüberschaubare Vielfalt an Gerüchen einer Systematik zu unterwerfen. Die Klassifizierung von Gerüchen ist eine fundamentale Voraussetzung dafür, den Geruchssinn zu verstehen, oder, nach Alexander Graham Bell (1914): „It is very obvious that we have very many different kinds of smells(...). But until you can measure their likeness and differences you can have no science of odour."

Bereits Aristoteles (384-322 vuZ) teilte Gerüche in sieben Kategorien ein. Ebenfalls sieben Kategorien schlug Carl von Linné (1707-1778), der schwedische Begründer der modernen Systematik, 1756 vor. Linné teilte die Gerüche in aromatici (würzig), fragrantes (wohlriechend), ambrosiaci (nach Moschus riechend), alliacei (nach Knoblauch riechend), hircini (nach Ziege riechend), tetri (abstoßend) und nausi (ekelerregend) ein. 1895 schlug der holländische Physiologe Hendrik Zwaardemaker (18571940) eine Klassifizierung in neun Gruppen vor (ätherisch, würzig, wohlriechend, ambrosiac (göttlich süß), alliaceous (nach Knoblauch riechend), faulig, hircine (nach Ziege riechend), empyreumatic (nach Verwesung riechend) und ekelerregend). Hans Henning stellte 1916 in dem Buch „Der Geruch“ die Legitimität der Geruchsklassifizierung Zwaardemakers, die auf subjektiven Geruchseindrücken einer einzelnen Person basierte, in Frage. Stattdessen entwikkelte Henning ein Geruchs-Prisma, bei dem die sechs Ecken des Prismas die Düfte faulig, fruchtig, harzig, brenzlich, würzig und blumig repräsentieren (Abbildung 2). Henning postulierte, dass jeder Geruch eine bestimmte Position in diesem dreidimensionalen Raum einnimmt und die Ähnlichkeit

Standardbeispiel dafür sind Benzaldehyde und Benzene, in denen die Aldehyd-Gruppe durch andere Gruppen ähnlicher Größe $\left(\mathrm{NO}_{2}, \mathrm{CN}\right.$ oder $\left.\mathrm{N}_{3}\right)$ ersetzt wurde. Benzaldehyd und dessen Derivate riechen alle nach bitteren Mandeln. Je ähnlicher die Form eines Moleküls der Form von Benzaldehyd ist, desto ähnlicher ist sein Geruch dem von bitteren Mandeln (Amoore 1971). Allerdings hat auch die, strukturell von Benzaldehyd vollkommen verschiedene, Blausäure $(\mathrm{HCN})$ den charakteristischen Mandelgeruch (Abbildung 3B). Die einzige Gemeinsamkeit zwischen Benzaldehyd und zweier Gerüche durch die Distanz zwischen ihnen repräsentiert wird. Auch Hennings Modell stellte kein akkurates Bild der Realität dar. 1927 entwickelten Crocker and Henderson ein Duft-KlassifizierungsSystem, das man käuflich erwerben konnte. Ihr System basierte auf vier Duftdimensionen (wohlriechend, säuerlich, brenzlich, caprylic (nach Ziege riechend)). Ein Duft wurde dadurch klassifiziert, dass ihm in jeder dieser vier psychologischen Dimensionen ein Wert von 1-8 zugeordnet wurde. Auch dieser Ansatz konnte sich nicht durchsetzen.

1962 stellte John Amoore eine Theorie vor, die auf Linus Paulings (1901-1994) Idee, dass die Form und Größe eines Moleküls für dessen Geruch verantwortlich ist, basierte. Diese Idee wiederum leitet sich von Emil Fischers (1852-1919) Postulat ab, nachdem Enzym und Substrat wie Schlüs-

sel und Schloss ineinander passen müssen, um zu interagieren. Nach Amoores System der Primärgerüche resultieren alle wahrgenommenen Gerüche aus einer Kombination von Primärgerüchen. Amoore glaubte, dass es bis zu 31 Primärgerüche gibt, identifizierte aber nur sieben. Für fünf davon wird der Geruch durch die Form des Moleküls bestimmt: nach Kampfer riechend (kugelig), nach Moschus riechend (scheibenförmig), blumig (rautenförmig), nach Pfefferminz riechend (keilförmig) and ätherisch (stabförmig). Zwei weitere Klassen von Gerüchen, stechend and faulig, würden sich nicht durch ihre Form, sondern durch die elektrische Ladung ihrer Teilchen auszeichnen. Obwohl sich Amoores Idee der Primärgerüche nicht durchsetzen konnte, ist die heute allgemein akzeptierte Theorie, dass der Geruch eines Moleküls durch seine Form bestimmt wird.

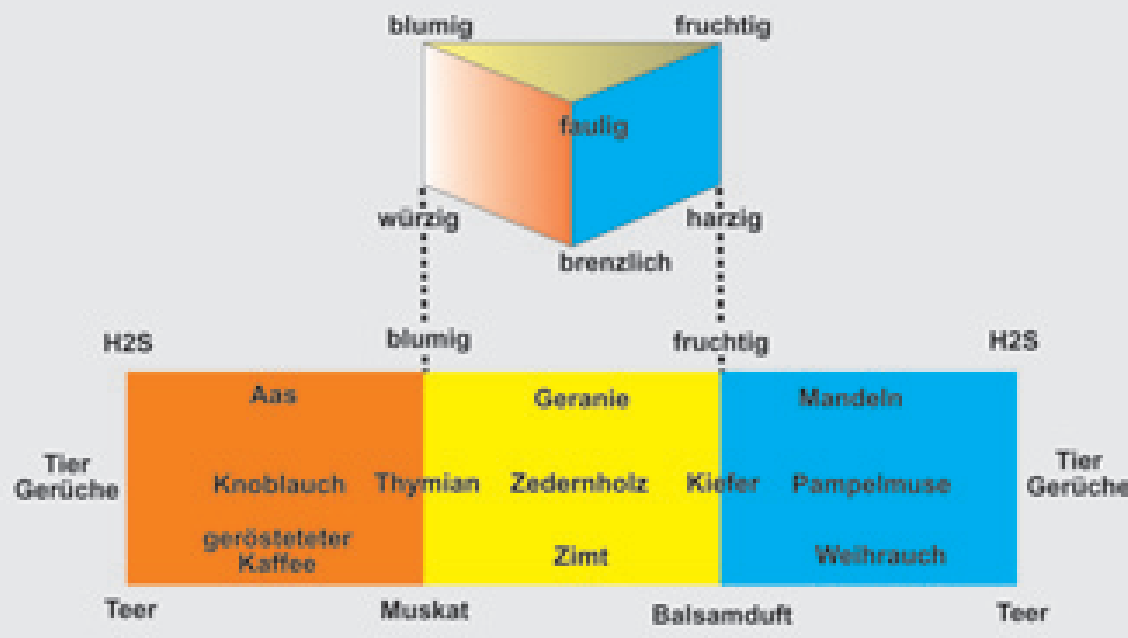

Abb. 2: Hennings Geruchs Prisma

Nach Henning lassen sich alle Düfte in einem dreidimensionalen Raum darstellen, der von einem Prisma begrenzt wird. Die sechs Ecken des Prismas repräsentieren die Düfte faulig, fruchtig, harzig, brenzlich, würzig und blumig. Jeder Geruch nimmt eine bestimmte Position in dem Prisma ein und ähnliche Gerüche liegen nahe beieinander. Hennings Theorie konnte sich nicht durchsetzen.

Blausäure ist, dass beide bei der Hydrolyse von Amygdalin, einem Bestandteil bitterer Mandeln, entstehen. Es wäre interessant zu sehen, ob diese beiden Düfte eine ähnliche Kombination von Riechzellen aktivieren, oder ob die wahrgenommene Ähnlichkeit lediglich auf einer Assoziation der beiden unterschiedlichen Duftmoleküle mit derselben Duftquelle, den Mandeln, beruht.

Eine Vielzahl von Molekülen mit extrem unterschiedlicher Form riechen nach Kampfer (Abbildung 3C). Einige Moleküle mit funktionellen Gruppen, die normalerweise mit unangenehmen Gerüchen verbunden werden (etwa Stickstoff, Phosphor oder Schwefel), riechen nach Kampfer. Aber auch Hydrokarbone ohne funktionelle Gruppe können nach Kampfer riechen. Der kleinste gemeinsame Nenner, auf den sich die Form der nach Kampfer riechenden Moleküle bringen lässt, ist, dass sie alle eiförmig sind und einen Durchmesser von etwa sieben Angström haben (Amoore 1970).

Diese und viele andere unterschiedlich erfolgreichen Versuche, den Geruch von Molekülen durch ihre Form zu erklären, sind ausführlich beschrieben wurden (Ros- 
siter 1996). In vielen Fällen kann der Sinneseindruck, den ein bestimmtes Duftmolekül hervorruft, nicht aufgrund der Form des Moleküls vorhergesagt werden, weswegen es sogar alternative Theorien gibt, die einen Zusammenhang zwischen der Form und dem Geruch eines Moleküls verneinen. Eine Theorie besagt, dass der Duft eines Moleküls nicht durch seine Form, sondern durch das Schwingungsverhalten der Elektronenbindungen bestimmt wird (Turin 1996). Allerdings gibt es auch für diese Annahme keinen überzeugenden Beweis. Die Ähnlichkeit der Gerüche vieler ähnlich geformter Moleküle spricht dafür, dass die Form eines Moleküls dessen Geruch beeinflusst. Der ähnliche Geruch verschiedenartig geformter Moleküle könnte dadurch zustande kommen, dass auch vollkommen verschiedene Kombinationen von aktivierten Riechzellen den gleichen subjektiven Geruchseindruck hervorrufen können. Dies kann beispielsweise der Fall sein, wenn zwei verschiedene Moleküle mit derselben Duftquelle assoziiert werden, wie im Fall der Mandeldüfte Benzaldehyd und Blausäure.

\section{Der Geruch eines Gemisches}

Nur in den seltensten Fällen werden wir den Geruch einer puren Chemikalie riechen. Viel häufiger besteht ein typischer Geruch aus einem Gemisch verschiedener Komponenten. Rosenöl etwa, das aus Rosenblüten extrahiert und zu Parfüms weiterverarbeitet wird, besteht aus etwa 275 verschiedenen Komponenten (Ohloff 1994). Das Unterscheiden sehr ähnlicher Geruchsgemische ist eine der wichtigsten Aufgaben des Geruchssinnes. Die Gerüche von frischer und schlechter Milch, reifen und faulen Früchten oder verschiedener Weine sind komplexe Geruchsgemische, die sich nur in einer oder wenigen Komponenten unterscheiden. Die Geruchseindrücke der Komponenten eines Gemisches werden vom Gehirn nicht einfach additiv zusammengefügt. Gemische ergeben in vielen Fällen eine völlig neue Geruchsgestalt, die sich dramatisch von der der Komponenten unterscheidet. In extremen Fällen kann das Gemisch zweier Gerüche nahezu geruchlos sein (Nagel 1897). In komplexen Gemischen können höchstens drei bis vier Komponenten identifiziert werden (Laing und Francis 1989). Überraschenderweise werden einzelne Gerüche subjektiv als gleich komplex wie Geruchsgemische wahrgenommen (Jellinek und Köster 1979) und im Gehirn wird durch einzelne Düfte ein ähnlich komple-
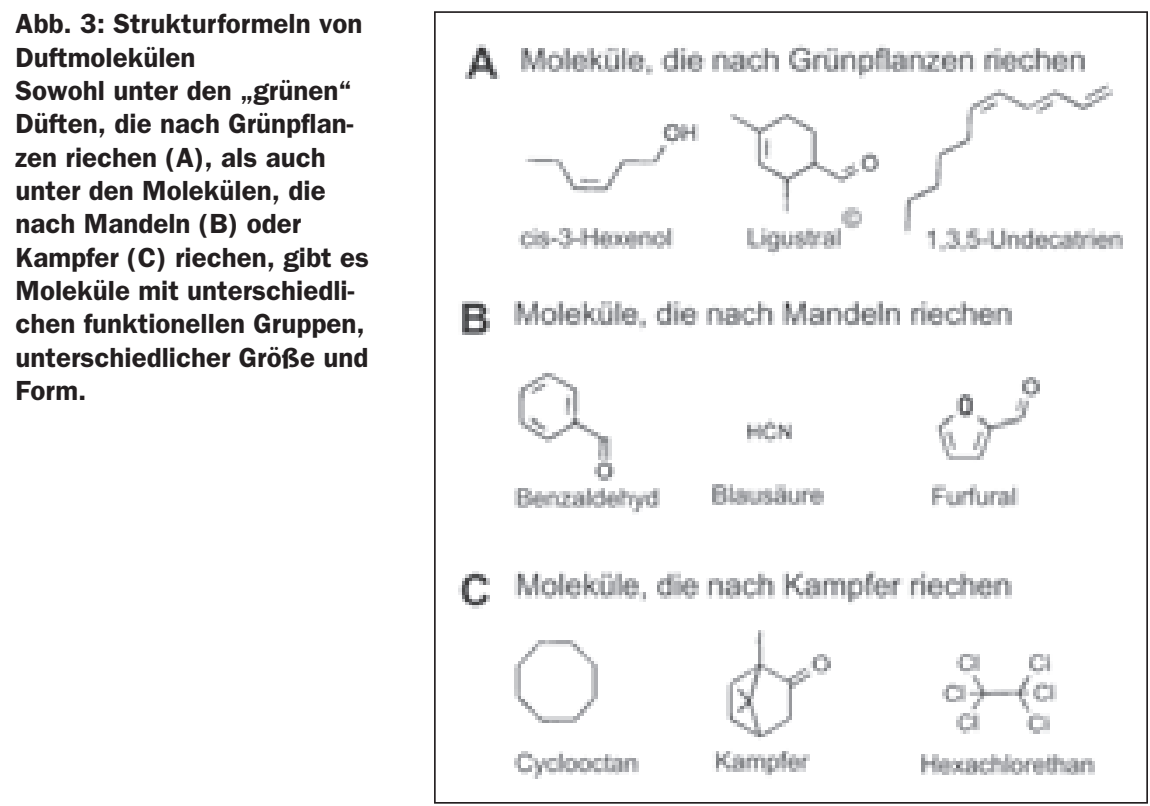

xes Muster von Neuronen angeregt, wie durch natürlich vorkommende Duftgemische (z.B. in Honigbienen (Apis mellifera): Galizia et al. 1999; Abbildung 5). All diese Beobachtungen deuten drauf hin, dass komplexe Duftgemische vom Gehirn als eine einzelne Geruchsgestalt, deren Geruch sich nicht direkt aus den Komponenten ableiten lässt, wahrgenommen werden. Die subjektive Bewertung von Geruchsgemischen als eine neue Geruchsgestalt kann durch zentralnervöse Prozesse beeinflusst werden. Warum Gemische kein komplexeres Muster von Neuronen anregen als einzelne Düfte kann jedoch nur durch Kompetition zwischen den verschiedenen Duftmolekülen eines Gemisches oder dadurch, dass aktive Neurone benachbarte Neurone inhibieren, erklärt werden. Der letztgenannte Mechanismus spielt nicht nur bei Duftgemischen, sondern auch bei der Verarbeitung einzelner Düfte eine Rolle (Wilson und Leon 1987).

\section{Die Anatomie des Geruchssinns}

Die Riechzellen im Riechepithel von Wirbeltieren (zwei Millionen in Mäusen) oder auf den Antennen und Maxillarpalpen von Insekten (1400 in der Fruchtfliege Drosophila melanogaster) werden von Duftmolekülen in der Luft aktiviert. Um durch ein Duftmolekül aktiviert zu werden, muss der Duftrezeptor in der Membran der Riechzelle mit dem Duftmolekül interagieren. Menschen haben etwa 350 verschiedene Duftrezeptoren, die mit unterschiedlichen Molekülen interagieren (Zozulya et al. 2001), Mäuse und der Nematode Caenorhabditis elegans haben über 1000 verschiedene Duftrezeptoren, die Fruchtfliege Drosophila melanogaster nur etwa 60. Es wird allgemein angenommen, dass jede Riechzelle nur einen bestimmten Rezeptor zum Erkennen von Duftmolekülen verwendet (aber siehe Exkurs: „Eine Riechzelle - ein Duftrezeptor - ein Glomerulus?"). Unterschiedliche nationen von Duftrezeptoren. Das daraus resultierende Aktivitätsmuster gibt Aufschluss über die Identität des Moleküls (Abbildung 1). Die Riechzellen, die den gleichen Rezeptor zur Dufterkennung verwenden, sind über das Riechepithel der Nasenschleimhaut (bei Insekten über die Antenne und in manchen Fällen über die Maxillarpalpen) verteilt. Die Axone der Riechzellen eines Typs konvergieren in der Regel alle in einer kugelförmigen Struktur, einem Glomerulus (Abbildung 4, aber siehe Exkurs: „Eine Riechzelle - ein Duftrezeptor - ein Glomerulus?"). Riechzellen bilden in den Glomeruli erregende Synapsen mit Ausgangsneuronen. Bei Wirbeltieren sind die Glomeruli im Riechkolben lokalisiert, bei Insekten im so genannten Antennallobus. Ein Duftmolekül interagiert mit verschiedenen Rezeptoren und aktiviert dadurch ein bestimmtes Muster an Glomeruli (Abbildung 5). Informationsaustausch zwischen den Glomeruli findet über lokale Neurone statt (Wirbeltiere: periglomeruläre Zellen und Körnerzellen; Insekten: lokale Interneurone). Aktive Glomeruli inhibieren das Signal in benachbarten Glomeruli, wodurch das Signal eines bestimmten Duftes verschärft wird. Es wurde vielfach gezeigt, dass die Aktivitätsmuster, die von Duftmolekülen Düfte aktivieren unterschiedliche Kombi- 


\section{Exkurs II}

\section{Eine Riechzelle - ein Duftrezeptor - ein Glomerulus?}

Das hier vorgestellte Modell des Riechsinns geht davon aus, dass jede Riechzelle nur einen Duftrezeptor zur Dufterkennung verwendet und dass die Axone eines Typs von Riechzellen in einem einzigen Glomerulus konvergieren. In den letzten Jahren hat es sich gezeigt, dass diese vereinfachende Vorstellung nicht immer richtig ist. Zuerst zeigten Vosshall et al. (2000), dass in Drosophila ein Duftrezeptor, Or83b, in der Mehrzahl der Riechzellen exprimiert wird. Diese Or83b-positiven Zellen exprimieren alle zusätzlich noch einen anderen Duftrezeptor, dessen Expression auf wenige Zellen beschränkt ist. Interresanterweise ist Or83b der einzige Duftrezeptor, der zwischen verschiedenen Insektenarten konserviert ist (Hill et al. 2002; Krieger et al. 2002). In einer Motte (Heliothis virescens) ist Or83b, wie in Drosophila, in der Mehrzahl der Riechzellen exprimiert (Krieger et al. 2002). In zumindest einer Riechzelle in Drosophila sind neben Or83b nicht nur einer, sondern zwei weitere Duftrezeptoren exprimiert (Dobritsa et al. 2003).

Auch in Wirbeltieren exprimiert nicht jede Riechzelle nur einen einzigen Duftrezeptor. Die Antworten von Riechzellen auf verschiedene Düfte sind oft am einfachsten mit der Existenz von zwei Duftrezeptoren in einer Riechzelle zu erklären (z.B. im Katzenwels: Kang und Caprio 1995). In Goldfischen wurde dann gezeigt, dass manche Duftrezeptoren, wie Or83b in Insekten, in einer Mehrzahl der Riechzellen exprimiert werden (Speca et al. 1999). Wahrscheinlich überlappen die Expressionsmuster und eine Goldfisch-Riechzelle exprimiert mehrere Duftrezeptoren. Der endgültige Beweis für das Vorhandensein mehrerer Duftrezeptoren in einer Riechzelle in Wirbeltieren gelang im Vomeronasal-Organ von Mäusen. Das Vomeronasal-Organ ist ein Riechorgan, das bei den meisten landlebenden Wirbeltieren eine groBe Rolle in der Pheromon-Kommunikation zwischen Individuen einer Art spielt. In den Riechzellen des Vomeronasal-Organs werden bei Mäusen zwei Duftrezeptoren exprimiert (Martini et al. 2001). Obwohl die Vielzahl dieser Befunde dar- auf hindeutet, dass ein Rezeptor pro Zelle eher die Ausnahme als die Regel ist, gibt es noch keine Studie über die Funktion der zusätzlichen Rezeptoren in einer Riechzelle. Es könnte sein, dass mehrere Rezeptoren direkt mit den Duftmolekülen interagieren. Alternativ könnte nur ein Rezeptor mit den Duftmolekülen interagieren, der oder die weiteren Rezeptoren aber an anderen Prozessen beteiligt sein. Denkbar wäre beispielsweise, dass der zweite Rezeptor an der Faltung und dem Transport des Rezeptors beteiligt ist oder, dass er zur Übermittlung des Signals notwendig ist.

In Drosophila konvergieren im Normalfall die Axone der Riechzellen, die gleiche Duftrezeptoren exprimieren, in einem Glomerulus im Antennallobus. Eine Ausnahme sind die Or23a-exprimierenden Riechzellen, die ihre Axone nicht in einen, sondern in zwei verschiedene Glomeruli senden (Vosshall et al. 2000). In einigen Individuen projizieren einige wenige Axone der verschiedensten Riechzellen-Typen sogar in mehr als zwei Glomeruli (Bhalerao et al. 2003).

In Mäusen projiziert ein Typ von Riechzellen in der Regel sowohl in den lateralen, als auch in den medialen Teil des Riechkolbens. Dort innervieren die Zellen normalerweise drei bis vier Glomeruli. Im Vomeronasal-Organ dagegen projizieren die Axone eines Typs von Riechzellen zu bis zu 30 Glomeruli (Belluscio et al. 1999; Rodrigues et al. 1999). Interresanterweise projizieren die Ausgangsneuronen des Riechkolbens ihre Dendriten zu mehreren Glomeruli, die alle vom gleichen Typ von Riechzellen innerviert werden (Del Punta et al. 2002). $\mathrm{Ob}$ man im Vomeronasal-Organ von Mäusen auch Glomeruli findet, in die verschiedene Typen von Riechzellen konvergieren, ist umstritten (Belluscio et al. 1999; Del Punta et al. 2002).

Einige grundlegende Annahmen des Modells in Abbildung 4 sind also zumindest nicht universell gültig. Bei einer immer größer werdenden Zahl von Riechzellen wird beschrieben, dass sie mehr als einen Rezeptor exprimiert und Riechzellen, die dieselben Rezeptoren exprimieren, können zu verschiedenen Glomeruli projizieren. Die Idee „Eine Riechzelle - ein Duftrezeptor - ein Glomerulus" ist wohl prinzipiell richtig, aber es gibt eine Reihe von Ausnahmen, deren Bedeutung für die Verarbeitung von Duftinformation noch unklar ist. mit ähnlicher Form hervorgerufen werden, sich nur wenig unterscheiden (z.B. in Honigbienen: Sachse et al. 1999). Die Ausgangsneurone (Wirbeltiere: Pinsel- und Mitral-Zellen; Insekten: Projektionsneurone) lesen die Information aus den Glomeruli aus und leiten sie an höhere Gehirnzentren weiter (Wirbeltiere: olfaktorischer Kortex; Insekten: Pilzkörper und Protocerebrum).

Die lokalen Interneurone, die benachbarte Glomeruli inhibieren können, ermöglichen es darüber hinaus, die Entladungen der Ausgangszellen zu synchronisieren. Diese zeitlichen Aktivitätsmuster sind ebenso wie die räumlichen Muster Duft-spezifisch (Laurent 1996). Die Information über die Identität eines Duftes könnte dem Gehirn also sowohl als räumliches als auch als zeitliches Aktivitätsmuster übermittelt werden. Erst Experimente, die es ermöglichen, das zeitliche Muster zu zerstören, das räumliche aber zu erhalten, könnten die Rolle der beiden Muster aufklären.

\section{Düfte und Duftrezeptoren}

Eine offensichtlich grundlegende Frage für das Verständnis des Geruchssinns ist, welche Duftmoleküle mit einem bestimmten Rezeptor interagieren. Da letztendlich der Duft eines Moleküls durch dessen Interaktionen mit Duftrezeptoren bestimmt wird, ist diese Frage eng mit der oben diskutierten Frage, was den Geruch eines Moleküls ausmacht, verbunden.

Es gibt verschiedene Ansätze, Duftmoleküle, die mit einem Rezeptor interagieren, zu identifizieren. Ein Duftrezeptor kann in einem heterologen System funktionell exprimiert werden (z.B. Krautwurst et al. 1998; Wetzel et al. 2001). Dafür wird beispielsweise ein Duftrezeptor artifiziell in einer Frosch-Eizelle exprimiert. Der Rezeptor wird in die Membran der Eizelle integriert und kann durch Interaktion mit einem Duftmolekül aktiviert werden. Die Aktivierung des Duftrezeptors resultiert in einem Stromfluss, der gemessen werden kann. Durch Applikation verschiedener Duftmoleküle werden diejenigen identifiziert, die den untersuchten Rezeptor aktivieren. Natürlich können die Bindungseigenschaften eines Duftrezeptors auch in einer intakten Riechzelle physiologisch untersucht werden. Eine weitere Möglichkeit ist es, die Antworten auf verschiedene Düfte in den Ausgangsneuronen der Glomeruli zu messen. Die Duft-induzierte neuronale Aktivität kann sowohl elektrophysiologisch als auch durch bildgebende Verfahren gemessen werden. Als besonders vielversprechend 
Abb. 4: Anatomie des Geruchssinns

(A) Duftmoleküle werden von einer bestimmten Gruppe von Duftrezeptoren in der Membran der Riechzellen gebunden. Riechzellen, die den gleichen Duftrezeptor exprimieren und daher die gleiche Bindungsspezifität haben, projizieren in einen Glomerulus. Lokale Neurone verbinden die Glomeruli und ermöglichen einen lateralen Informationsaustausch. Ausgangsneurone leiten die Duftinformation von den Glomeruli weiter in höhere Gehirnregionen. Dieses Schema zeigt das grundlegende Prinzip, wie Geruchsinformation sowohl in Wirbeltieren, als auch in Insekten, verarbeitet wird.

(B) Die zwei Antennalloben von Drosophila sind in einer Dreifachfärbung gezeigt. Alle Neurone, einschliesslich der Antennalloben (AL) sind rot gefärbt. Riechzellen, die den Duftrezeptor Or22a exprimieren, sind grün gefärbt. Die Zellkerne, Axone und Dendriten der Riechzellen liegen in der Antenne und sind hier nicht zu sehen, alle übrigen Zellkerne sind blau gefärbt. Die Innervation der Riechzellen sind in dem entsprechenden Glomerulus (DM2) des Antennallobus zu sehen (Pfeil).

(C) Zwei Antennalloben von Drosophila sind gezeigt. Einige lokale Interneurone, die Glomeruli miteinander verbinden, sind grün gefärbt, die anderen Neurone sind wieder in rot dargestellt.

(D) Ein Drosophila Gehirn ist gezeigt. Man sieht in rot den Optischen Lobus (OL) und das Zentralgehirn. In grün sind einige Projektionsneurone gefärbt. Projektionsneurone sind Ausgangsneurone, die den Antennallobus mit dem Pilzkörper (PK) und dem Protocerebrum (PC) verbinden. (Balken: $50 \mu \mathrm{m}$ )

(Abbildungen B, C und D freundlichst zur Verfügung gestellt von Dr. Silke Sachse, New York)

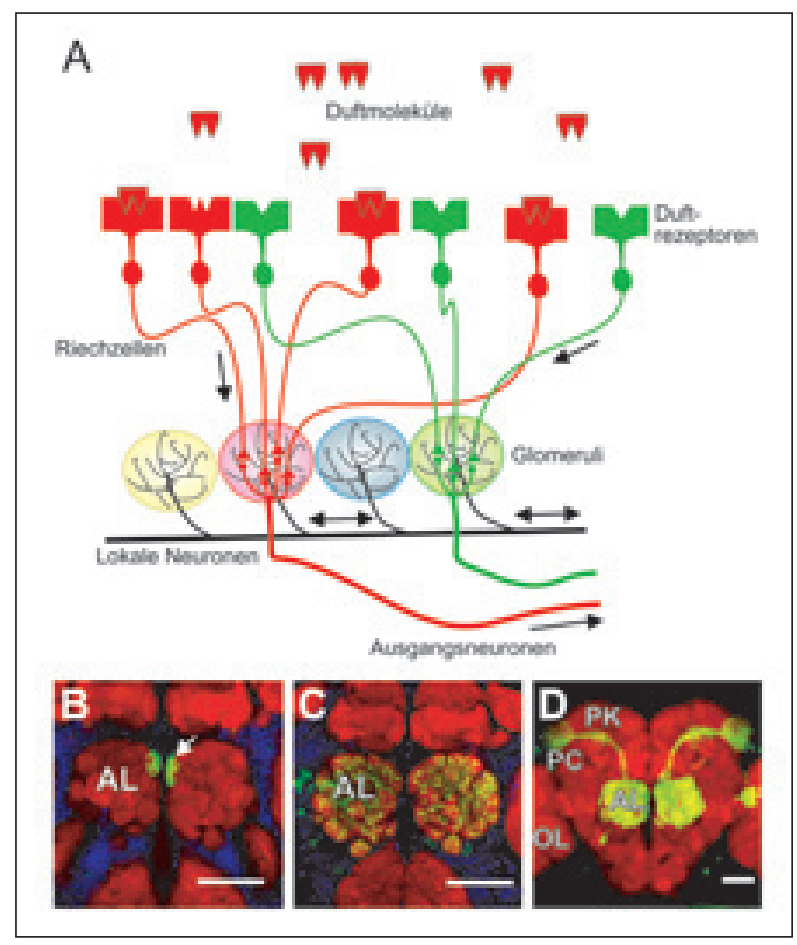

hat sich dabei das Messen der neuronalen Antwort mit Hilfe von transgenen KalziumIndikatoren erwiesen. Kalzium-Indikatoren können zellspezifisch exprimiert werden und ermöglichen es, den Anstieg der Kalziumkonzentration in aktiven Neuronen zu visualisieren.

Eine Arbeit, in der die Interaktionen eines Duftrezeptors aus der Ratte mit 90 verschiedenen Duftmolekülen untersucht wurde, zeigte, dass der untersuchte Rezeptor [I7] ausschließlich mit Aldehyden interagierte (Araneda et al. 2000). Interessanterweise reagierte der Rezeptor jedoch nicht mit allen getesteten Aldehyden. Citronellal aktivierte den Rezeptor stark, während Citral, ebenfalls ein Aldehyd, den Rezeptor nicht aktivierte. Citronellal und Citral riechen beide nach Limonen und unterscheiden sich in ihrer Struktur lediglich durch eine zusätzlich Doppelbindung im Citral. Auch elektrophysiologische Messungen an Ausgangsneuronen der Glomeruli in Hasen ergaben keine eindeutigen Ergebnisse. Die Gesamtstruktur des Duftmoleküls sowie die Art und Position der funktionellen Gruppe beeinflussten die Interaktion zwischen Rezeptor und Duftmolekül, allerdings konnten auch hier keine eindeutigen Regeln gefunden werden, mit denen sich eine erfolgreiche Interaktion vorhersagen lassen könnte (Imamura et al. 1992; Katoh et al. 1993).

Die Experimente mit Riechzellen aus Wirbeltieren haben den Nachteil, dass der sehr komplexe Geruchssinn der Wirbeltie- re aus hunderten verschiedener Duftrezeptoren und Riechzellen besteht. Da transgene Wirbeltiere nur mit großem Aufwand herzustellen sind, wäre eine Charakterisierung der Bindungseigenschaften aller Duftrezeptoren eines Wirbeltiers ein sehr um- fangreiches Unterfangen. Im Gegensatz dazu eignet sich die genetisch einfach $\mathrm{zu}$ manipulierende Fruchtfliege Drosophila melanogaster mit nur etwa 60 Duftrezeptoren hervorragend als Modell zur Erforschung des Geruchssinns. Die Organisation
Abb. 5: Aktivierungsmuster von Nelkenöl, einem natürlich vorkommenden komplexen Gemisch, und Oktanol, einem Alkohol, im Antennallobus der Honigbiene Apis mellifera.

Mit Hilfe von bildgebenden Verfahren wurde die Kalziumkonzentration als Indikator für neuronale Aktivität gemessen. In (A) ist ein Beispiel einer Aktivitätsmessung während Nelkenöl (oben) oder Oktanol (unten) Stimulierung zeigt. Die glomeruläre Struktur des Antennallobus ist darüber projeziert. Die Kalziumkonzentration ist in Falschfarben repräsentiert, d.h. Rot/Gelb stellen eine Erhöhung der Kalziumkonzentration dar, während Blau für eine unveränderte Konzentration steht. In (B) wurden die Kalziumaktivitäten wie die in (A) gezeigte von mehreren Bienen gemittelt und in einem standardisierten Antennallobus dargestellt (Nelkenöl: $\mathbf{n = 5}$; Oktanol: $n=21$ ). Nelkenöl und Oktanol aktivieren verschiedene Muster von Glomeruli. Nelkenöl aktiviert Glomerulus 47 und 60 am stärksten, während Oktanol Glomerulus 17 sehr stark aktiviert. (Abbildung freundlichst zur Verfügung gestellt von Dr. Silke Sachse, New York)

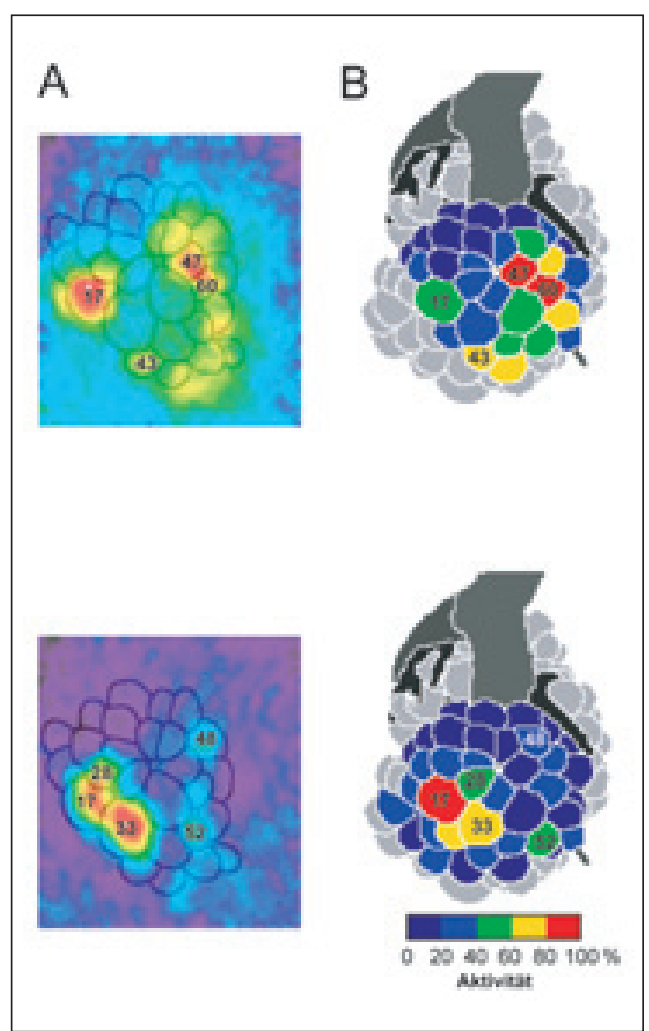




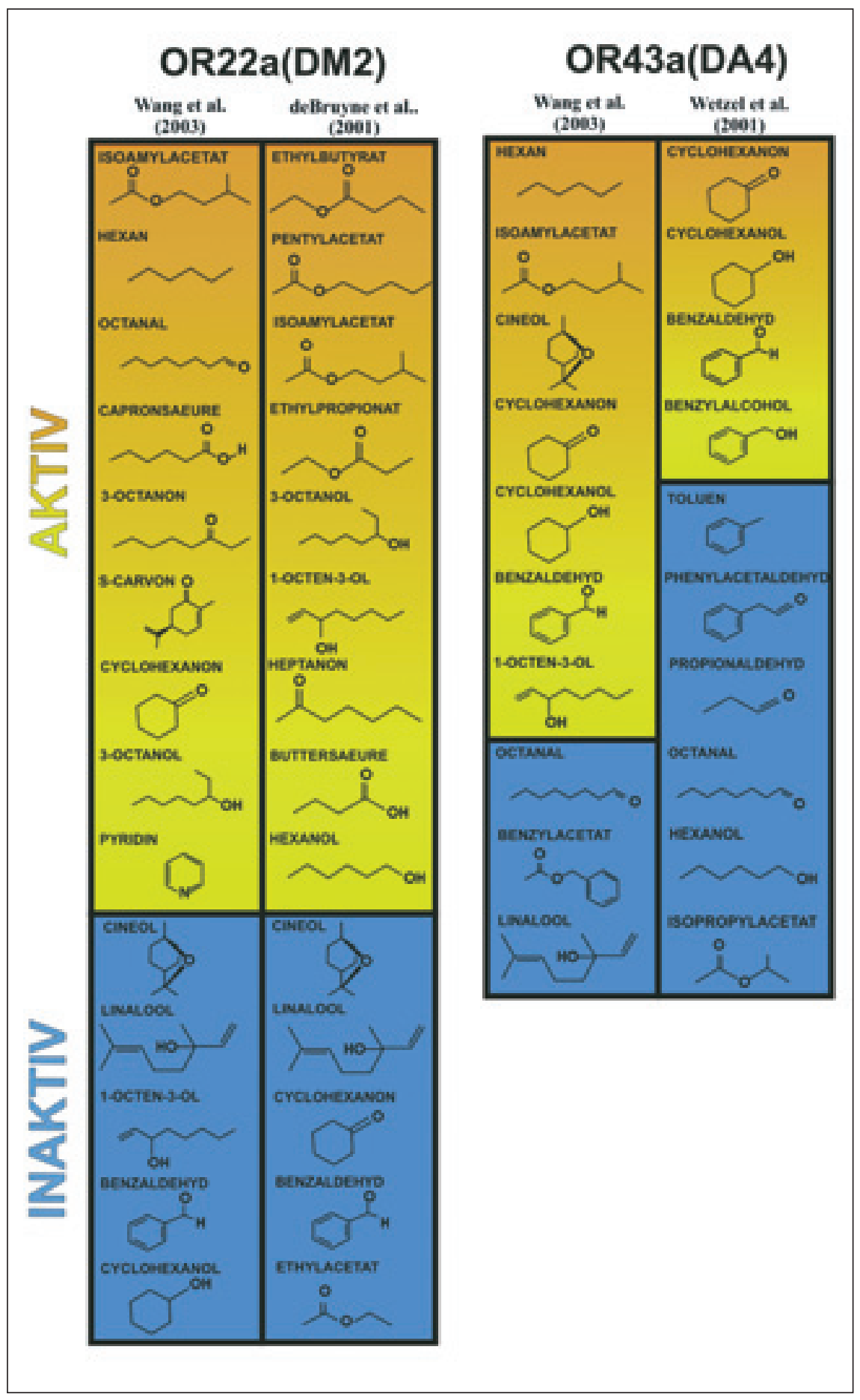

Abb. 6: Duftrezeptoren und Duftmoleküle

Die Moleküle, die mit dem Drosophila-Duftrezeptor Or22a beziehungsweise Or43a interagieren, wurden auf jeweils zwei verschiedene Arten bestimmt. Die Axone Or22a exprimierender Riechzellen konvergieren im Glomerulus DM2, die Axone Or43a exprimierender Riechzellen in DA4. Wang et al. (2003) bestimmte mit Hilfe bildgebender Verfahren aus 16 Düften diejenigen, die mit Or22a und Or43a interagierten. De Bruyne et al. (2001) bestimmte durch extrazelluläre Ableitungen die Reaktion von Riechzellen auf 47 Düfte. Wetzel et al. (2001) testete 13 Düfte auf ihre Interaktion mit dem Rezeptor in einem heterologen System. Hier ist nur eine Auswahl der Düfte gezeigt. Alle Düfte, die in beiden der verglichenen Studien verwendet wurden, sind gezeigt. Die aktiven Düfte sind nach der Stärke der Aktivierung geordnet. des Geruchssinns der Fliegen ist detailliert beschrieben (Keller und Vosshall 2003) und der in anderen Insekten und Wirbeltiere sehr ähnlich. Es kann erwartet werden, dass Experimente in Drosophila in allgemeingültigen Erkenntnissen resultieren. Die Voraussetzung für eine Identifizierung von Interaktionspartnern der Drosophila - Duftrezeptoren war deren Identifizierung und Klonierung, die sich wegen deren geringer Homologie als schwierig erwies und erst 1999 gelang (z.B. Vosshall et al. 1999). Seitdem wurden mit einer Reihe verschiedener Methoden Düfte identifiziert, die mit bestimmten Rezeptoren interagieren (de Bruyne et al. 1999; de Bruyne et al. 2001; Wetzel et al. 2001; Dobritsa et al. 2003; Pelz et al. 2003; Stensmyr et al. 2003; Wang et al. 2003). In Abbildung 6 sind Ergebnisse, die mit Hilfe verschiedener Techniken erzielt wurden, verglichen. Die verglichenen Studien kommen für Düfte, die in beiden Studien verwendet wurden, zu sehr ähnlichen Ergebnissen.

Interessant ist, dass jeder der beiden Rezeptoren in Abbildung 6 von sehr unterschiedlichen Molekülen aktiviert wurde. Or22a interagierte mit Estern, Aldehyden, Ketonen, Aromaten, Säuren und einem Hydrokarbon ohne funktionelle Gruppe. Die Interaktionspartner von Or22a rufen die unterschiedlichsten Geruchseindrücke hervor. Buttersäure riecht nach ranziger Butter, Isoamylazetat nach Banane und s-Carvon nach Kümmel. Darüber hinaus war Ethylbutyrat der beste Interaktionspartner für Or22a, während das strukturell sehr ähnliche Ethylazetat überhaupt nicht interagierte (Abbildung 6). Beide Studien kommen zu dem Ergebnis, dass Or22a mit vielen strukturell verschiedenen Düften interagiert. $60 \%$ beziehungsweise $30 \%$ der getesteten Düfte aktivierten Or22a. In einer dritten Studie, in der 93 Düfte getestet wurden, induzierten ebenfalls 30\% der Düfte eine Antwort (Pelz et al. 2003). Or22a ist kein Sonderfall, andere Rezeptoren sind sogar noch unspezifischer (de Bruyne et al. 2001; Wang et al. 2003). Interessanterweise wurden aber auch Rezeptoren gefunden, die unter den gleichen experimentellen Bedingungen nur auf einen einzelnen oder auf keinen der getesteten Düfte reagierten. Auch in einer Studie, in der nur ökologisch relevante Düfte von überreifen oder faulen Früchten getestet wurden, zeigten mehr als ein Drittel der getesteten Riechzellen auf keine der 23 Düfte eine Reaktion (Stensmyr et al. 2003). Es scheint, als reagierten nicht alle Riechzellen auf ein ähnlich breites Spektrum von Düften. Vielmehr gibt es spezialisierte Riechzellen, deren Interak- 
tionspartner noch nicht identifiziert werden konnten. Eine andere Klasse von Riechzellen dagegen reagiert relativ unspezifisch auf viele strukturell unterschiedliche Düfte.

\section{Die drei grundlegenden Fragen}

Welche Eigenschaften eines Duftmoleküls sind für die Interaktion mit einem Rezeptor entscheidend? Mit der Identifizierung und Klonierung der Drosophila-Duftrezeptoren wurde ein Modellsystem mit einem überschaubar komplexen Geruchssinn experimentell zugänglich gemacht. Die Kombination von bildgebenden Verfahren zur Visualisierung neuronaler Aktivität und transgener Methoden wird sicher in naher Zukunft dazu beitragen, Interaktionspartner für die meisten Drosophila-Duftrezeptoren zu identifizieren. Die Analyse dieser Daten wird hoffentlich Klarheit darüber schaffen, welche Eigenschaften der Duftmoleküle für die Interaktion mit einem Rezeptor ausschlaggebend sind.

Welche Eigenschaften eine Duftmoleküls sind für den subjektiven Geruchseindruck entscheidend? Solange der Unterschied zwischen zwei Gerüchen nicht physikalisch bestimmt oder errechnet werden kann, sind psychophysikalische Experimente die einzige Möglichkeit, diese Frage zu beantworten. Neue Theorien, deren Vorhersagen getestet werden können, sind dringend notwendig, um endlich soweit zu kommen, dass der Geruch eines Moleküls zuverlässig vorhergesagt werden kann.

Worin besteht der Zusammenhang zwischen der Interaktion des Duftmoleküls mit den Duftrezeptoren und dem letztendlich daraus resultierenden Geruchseindruck? Dieser Zusammenhang könnte durch eine Kombination von Aktivitätsmessung in Riechzellen in einem Modellorganismus und psychophysikalischer Experimente untersucht werden. Dafür müsste man aber davon ausgehen, dass z.B. Benzaldehyd und Blausäure, die für uns beide nach bitteren Mandeln riechen, auch für eine Fliege ähnlich riechen. Will man diese Annahme nicht machen, muss man Verhaltensexperimente (Verhalten wird vom Geruchseindruck gesteuert, nicht von dem Aktivitätsmuster der Rezeptorneurone) in einem Modellorganismus mit der Aktivitätsmessung in Rezeptorneuronen kombinieren.

\section{Literatur}

Araneda, R.C., Kini, A.D. und Firestein, S. (2000): The molecular receptive range of an odorant receptor. Nat. Neurosci. 3: 1248-1255.
Buck, L. und Axel, R. (1991): A novel multigene family may encode odorant receptors a molecular basis for odor recognition. Cell 65: 175-188.

Ohloff, G. (1994): Scent and Fragrances: The Fascination of Odors and their Chemical Perspectives. Berlin: Springer-Verlag; 154-158.

Rossiter, K.J. (1996): Structure-Odor relationships. Chem. Rev. 96: 3201-3240.

Wang, J.W., Wong, A.M., Flores, J., Vosshall, L.B., Axel, R. und Shigeo, J.F. (2003): Twophoton calcium imaging reveals an odor-evoked map of activity in the fly brain. Cell 12: 271-282.

Eine vollständige Literaturliste kann beim Autor angefordert werden.

\section{Danksagung}

Mein Dank gilt Silke Sachse und Leslie Vosshall für die Unterstützung. Die Forschungsarbeiten des Autors werden gegenwärtig vom NIH (5R01DC005036-03) und der Marco S. Stoffel Fellowship in Mind, Brain and Behavior gefördert.

\section{Kurzbiographie}

Andreas Keller, geboren 1972 in Nürnberg, studierte an der Friedrich-Alexander-Universität in Erlangen Biologie. Promotion 2002 am Lehrstuhl für Genetik und Neurobiologie der Julius-Maximilians-Universität in Würzburg in der Arbeitsgruppe von Professor Heisenberg. Seit 2002 im Laboratory of Neurogenetics and Behavior der Rockefeller University in New York (USA) in der Arbeitsgruppe von Professor Vosshall.

\section{Korrespondenzadresse}

\section{Dr. Andreas Keller}

Rockefeller University

Box 63

1230 York Avenue

NY, NY 10021

United States of America

Tel.: 001-212-327-7269

Fax: 001-212-327-7238

e-mail:kellera@mail.rockefeller.edu

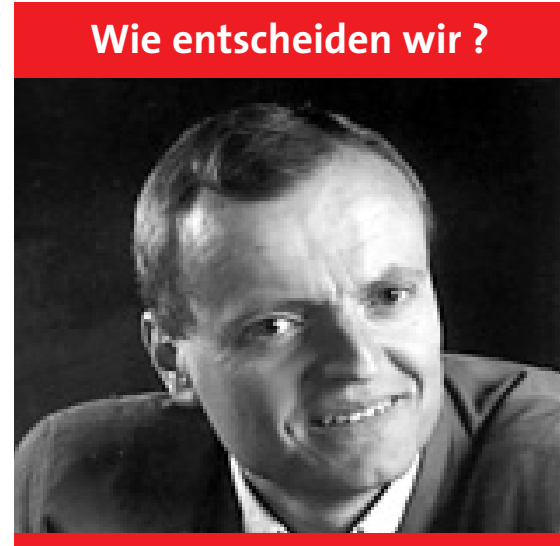

Antworten vom Erfolgsautor Manfred Spitzer!

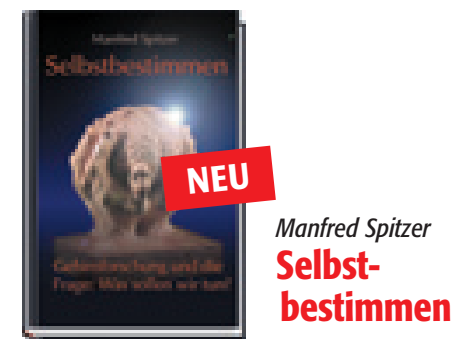

Manfred Spitzers neuestes Buch Selbstbestimmen handelt von der Frage: Wie entscheiden wir uns? Was treibt uns beim Handeln an? Kurz: Wie bestimmen wir, was wir tun und vor allem: Was sollen wir tun?

Nur wenn wir verstehen, warum wir was ohnehin dauernd tun und welche Fehler wir dabei machen, im Denken und im Handeln, haben wir eine Chance, die Frage danach, was wir tun sollen, sinnvoll und besser als bisher zu beantworten.

ca. 400 S., HC; $€ 29,95$, ISBN 3-8274-1489-X Ersch.-Termin: Ende Nov.2003 -bitte jetzt vormerken lassen!

\section{Bereits über 25.000 verkaufte Exemplare}

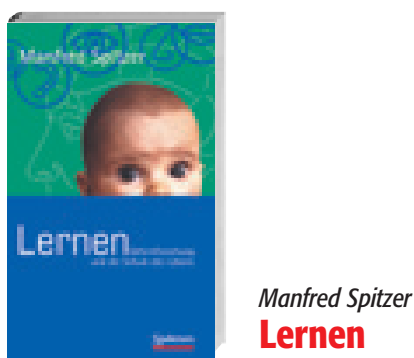

Lernen ist die natürliche und nicht zu bremsende Lieblingsbeschäftigung unseres Gehirns. Wie unsere "Lernmaschine im Kopf" arbeitet und wie wir sie mit Lernerfolg - und auch Vergnügen - arbeiten lassen können, das vermittelt dieses spannende Buch. 2002, 500 S., 93 Abb., geb.; $€ 29,95$, ISBN 3-8274-1396-6

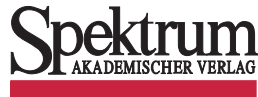

Bestellen können Sie

a telefonisch $07071 / 935369$ a per Fax 07071/935393

口 per Mail: shop@spektrum-verlag.de 